$12-15-1987$

\title{
Theory of the Observations Made of High-Order Rainbows from a Single Water Droplet
}

James A. Lock

Cleveland State University, j.lock@csuohio.edu

Follow this and additional works at: https://engagedscholarship.csuohio.edu/sciphysics_facpub

Part of the Physics Commons

How does access to this work benefit you? Let us know!

\section{Publisher's Statement}

This paper was published in Applied Optics and is made available as an electronic reprint with the permission of OSA. The paper can be found at the following URL on the OSA website: http://www.opticsinfobase.org/ao/abstract.cfm?URI=ao-26-24-5291.

Original Citation

Lock, James A. "Theory of the Observations Made of High-Order Rainbows from a Single Water Droplet." Applied Optics 26 (1987): 5291-5298.

\section{Repository Citation}

Lock, James A., "Theory of the Observations Made of High-Order Rainbows from a Single Water Droplet" (1987).

Physics Faculty Publications. 137.

https://engagedscholarship.csuohio.edu/sciphysics_facpub/137

This Article is brought to you for free and open access by the Physics Department at EngagedScholarship@CSU. It has been accepted for inclusion in Physics Faculty Publications by an authorized administrator of

EngagedScholarship@CSU. For more information, please contact library.es@csuohio.edu. 


\title{
Theory of the observations made of high-order rainbows from a single water droplet
}

\author{
James A. Lock
}

\begin{abstract}
Over a dozen rainbows have been observed in a single water droplet. They appear as glare spots on the water droplet which take on coloration at the appropriate rainbow angles. The appearance of rainbows as colored glare spots in this situation is understood in terms of the caustics created in the vicinity of the droplet by the refracting light rays. The angular positions of the glare spots are understood in terms of the Fourier transform of the geometric scattering amplitude. The rainbow glare spots are also found to appear numerically in the Fourier transform of the Mie scattered fields. An additional glare spot produced by rays at grazing incidence and not attributable to geometric optics also appears numerically in the Fourier transformed Mie fields.
\end{abstract}

\section{Introduction and Qualitative Treatment}

Despite the occasional accounts of the observation of the third-order rainbow, ${ }^{1}$ it is commonly believed that only the first- and second-order rainbows are visible in the atmosphere. ${ }^{2,3}$ This belief is supported, at least for spherical raindrops, by Mie scattering calculations in which the first- and second-order rainbows along with their supernumeraries prominently appear in the scattered intensity, but the third- and fourth-order rainbows are buried in the forward hemisphere diffracted and/or directly transmitted light. ${ }^{4-8}$ The situation is quite different for observations made on a single water droplet where at least thirteen rainbows have been seen. ${ }^{9-11}$ The rainbows observed from a single water droplet do not appear as arcs of color in the sky but rather as glarelike spots of color on the surface of the droplet itself. It is the purpose of this paper to understand the formation of these rainbow glare spots using Mie scattering theory and the results of the complex angular momentum theory for high frequency scattering.

In the Descartes ray tracing theory of the rainbow, parallel rays of sunlight enter a water droplet and are reflected within it a number of times before leaving the droplet. The angular deviation of an incident ray

The author is with Cleveland State University, Physics Department, Cleveland, Ohio 44115.

Received 7 May 1987.

0003-6935/87/245291-08\$02.00/0.

(C) 1987 Optical Society of America. caused by this refraction-multiple reflection-refraction process possesses a relative maximum or minimum for one of the rays. This is known as the rainbow ray, and its exit angle is the angle at which the rainbow occurs in the Descartes theory. To one side of the angle is a shadow region into which no rays emerge. The superposition of the shadow regions associated with the first- and second-order rainbows forms the well-known Alexander's dark band. To the other side of the rainbow angle is a lit region where the scattered rays fold back over on themselves producing the supernumerary interference pattern adjacent to the main rainbow.

Tricker and Walker have observed and described the appearance of a rainbow from single droplet of water as the observer moves from the lit region on one side of the rainbow angle to the shadow region on the other side. ${ }^{12}$ They find that in the lit region far from the rainbow angle there is a single white glare spot, hereafter called $R_{a}$, seeming to originate on the rear surface of the droplet. As the observer moves toward the rainbow angle, $R_{a}$ moves along the rear surface of the droplet toward the edge. At some observer angle another white glare spot, hereafter called $R_{b}$, appears on the edge of the droplet. As the observer continues toward the rainbow angle, $R_{a}$ and $R_{b}$ move toward each other, $R_{a}$ along the rear surface of the droplet and $R_{b}$ along the front surface. On moving through the rainbow angle, $R_{a}$ and $R_{b}$ merge and take on color, at first blue, then green, yellow, orange, and finally red. As the observer moves into the shadow region, the glare spots disappear.

In addition, there are brighter glare spots on the surface of the droplet due to rays directly reflecting from the surface $G$ and rays transmitted through the 


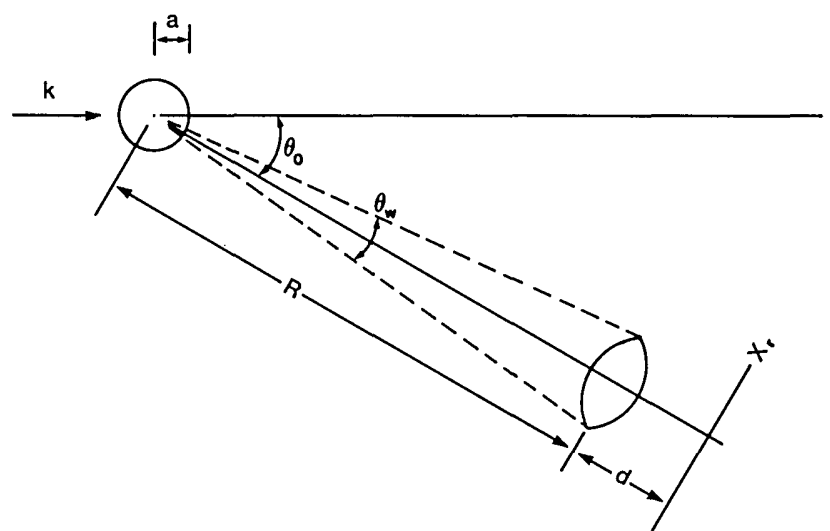

Fig. 1. Observer's eye is a distance $R$ from the water droplet of radius $a$ and is centered at the scattering angle $\theta_{0}$. The eye subtends an angle $\theta_{w}$, and the coordinate on the retina a distance $d$ behind the eye lens is $x_{r}$.

droplet and partially focused by it $T$. These brighter glare spots often partially obscure the visibility of the rainbow glare spots $R_{a}$ and $R_{b}$. This partial obscuring occurs for the third- and fourth-order rainbows and has been credited as being one cause of their supposed invisibility in the atmosphere.

These observations are qualitatively understood in terms of the caustic surfaces formed in the neighborhood of the droplet by the exiting light rays and in terms of the focusing properties of the eye. Half of the exiting rays in the lit region form a real caustic beginning near the droplet surface and ending on the rainbow ray. ${ }^{13}$ The other half of the rays if traced backward forms a virtual caustic beginning on the rear side of the droplet and extending infinitely far back from it with the extension of the rainbow ray as an asymptote. Because the caustics are the envelopes of the virtual or real intersection points of the light rays reaching the observer in the lit region, points on the caustics act as the virtual or real sources of the glare spots $R_{a}$ and $R_{b}$. As the observer moves through the lit region, the source points move along the caustics. Since the caustics begin on the droplet, the glare spots are usually observed as being on or near the droplet as well. If the observer is far enough into the lit region, rays from only the virtual caustic reach him, and he sees only the $R_{a}$ glare spot. As he approaches the rainbow angle, both caustics approach the same asymptote, and as a result the sources of the two glare spots approach the same line of sight.

To determine the role of the focusing properties of the eye on the observation of high-order rainbows from a droplet of water, consider the situation where the size of the eye is small enough so that as the observer moves from the lit region to the shadow region of a high-order rainbow, the scattered light reaching him is approximately confined to a single plane. If the observer stands at the scattering angle $\theta_{0}$ a distance $R$ from the droplet of radius $a$, and the pupil of the eye subtends an angle $\theta_{w}$ as in Fig. 1, the electric field at a position $x_{r}$ on the retina of a well-focused eye is

$$
\mathbf{E}_{r}\left(x_{r}\right)=\int_{-\theta_{w / 2}}^{\theta_{w / 2}} d \xi \mathbf{E}_{\mathrm{Mie}}\left(\theta_{0}+\xi\right) \exp \left(-i k R \xi x_{r} / d\right),
$$

where $\mathbf{E}_{\text {Mie }}$ is the Mie scattered electric field, $d$ is the eye lens-retina separation, and

$$
k=(2 \pi) / \lambda \text {. }
$$

This may be rewritten as the Fourier transform of the Mie electric field incident on the eye lens convolved with the Fourier transform of the lens's aperture function

$$
S(\xi)= \begin{cases}1 & \text { if }|\xi| \leq \theta_{w / 2}, \\ 0 & \text { if }|\xi|>\theta_{w / 2}\end{cases}
$$

and takes the form

$$
\mathbf{E}_{r}(p)=\int_{-\infty}^{\infty} d \alpha F_{s}(\alpha) \mathbf{F}_{\mathrm{Mie}}\left(p-\alpha, \theta_{0}\right),
$$

where

$$
\begin{aligned}
p & =k R x_{r} / d, \\
F_{s}(\alpha) & =\theta_{w} \operatorname{sinc}\left(\frac{\alpha \theta_{w}}{2}\right), \\
\mathbf{F}_{\mathrm{Mie}}\left(\alpha, \theta_{0}\right) & =\int_{-\infty}^{\infty} d \xi \mathbf{E}_{\mathrm{Mie}}\left(\theta_{0}+\xi\right) \exp (-i \alpha \xi) .
\end{aligned}
$$

If the pupil subtends a large angle $\theta_{w}$ as would be the case when examining a single water droplet at a close distance, $F_{s}(\alpha)$ approaches a $\delta$-function, and the observed field becomes $\mathbf{F}_{\text {Mie }}\left(p, \theta_{0}\right)$ only minimally blurred by the wide pupil aperture. On the other hand, if the observer is a great distance away from the water droplets fa!ling in a rain shower, $\theta_{w} \rightarrow 0$, the pupil diffractive effects spread and completely obscure the structure of $\mathbf{F}_{\text {Mie }}\left(p-\alpha, \theta_{0}\right)$, and the observed field becomes proportional to $\mathbf{E}_{\mathrm{Mie}}\left(\theta_{0}\right)$. Thus, when the observer is far from the droplet, he sees the Mie scattered intensity. On the other hand, when he is close to the droplet, he sees the square of the Fourier transform of the Mie scattered electric field.

This may be applied to the situation when the observer is standing in the supernumerary region of a rainbow. As seen above, in this region there are two effective light sources located on the real and virtual caustics. Their interference pattern at the position of the observer has a sinusoidal spatial periodicity. This is the supernumerary interference pattern. Its spatial variation is slow close to the rainbow and is rapid far from the rainbow. 5,14 The eye performs a Fourier transform of the field incident on it and converts the supernumerary interference pattern into its two component spatial frequencies, widely separated far from the rainbow and close together near the rainbow. These two spatial frequencies are the glare spots, $R_{a}$ and $R_{b}$, the images of the effective sources on the caustics. In this region the spatial distribution of the observed glare spots is both the image of the effective sources and the Fourier transform of the source's interference pattern at the eye lens.

The Fourier transforming property of the eye may also provide a reason for the occasional reported obser- 
vation of the third-order rainbow in the atmosphere. In the Mie scattered intensity, the third-order rainbow signal is buried in the much stronger forward hemisphere diffractive peak, which we can consider to be noise. However, since the third-order rainbow signal and the diffractive noise have different spatial periodicities, they become separated in the Fourier domain. This separation of the rainbow glare spots $R_{a}$ and $R_{b}$ from the reflected and transmitted glare spots $G$ and $T$ is the reason that higher-order rainbows are visible in a single droplet of water and may contribute to their possible visibility in a nearby rain shower. ${ }^{15}$

One can also relate these observations to the photodetector signals obtained in the operation of a polar nephelometer. ${ }^{16}$ This device employs receiving optics to focus the scattered light intensity obtained over a certain angular interval onto a photocell. Parseval's theorem for conservation of energy applied to the Fourier transform pair $f(x)$ and $F(p)^{17}$ gives

$$
\int_{-\infty}^{\infty} \frac{d p}{2 \pi}|F(p)|^{2}=\int_{-\infty}^{\infty} d x|f(x)|^{2}
$$

Because the photocell records only the signal integrated over its sensitive area and not the functional dependence of the signal along the sensitive area, the photocell signal is proportional to the Mie intensity integrated over the angular extent of the receiving optics independent of whether the detector is near or far from the source of the scattered light. ${ }^{18}$

\section{Glare Spots and the Complex Angular Momentum Theory for High-Frequency Scattering}

In the limit $a / \lambda \gg 1$, each partial wave of the Mie infinite series expansion of the electric field scattered from a sphere may itself be rewritten as an infinite series of interactions of light rays with the surface of the sphere. The double series may then be rearranged as

$$
\mathbf{E}_{\mathrm{Mie}}(\theta)=\sum_{m=0}^{\infty} \mathbf{E}_{m}(\theta),
$$

where $m+1$ is the number of times a light ray reflects or refracts from the sphere surface. The $m=0$ term corresponds to direct reflection, $m=1$ to transmission, and $m \geq 2$ to multiple reflections within the sphere which produce rainbows. Each $\mathbf{E}_{m}(\theta)$ term can be converted from a sum over partial waves to an integral over the impact parameter. Each resulting integrand is dominated by a number of saddle points and poles in the complex plane. The saddle point contributions to the integrals correspond to geometric rays, and the residue pole contributions correspond to diffractive effects produced by surface waves. ${ }^{19,20}$ The saddle point contribution to $\mathbf{E}_{m}(\theta)$ is of the form

$$
\mathbf{E}_{m}^{(g)}(\theta)=\mathbf{A}_{m}(\theta) \exp \left[i \phi_{m}(\theta)\right],
$$

where $\mathbf{A}_{m}(\theta)$ is the magnitude of the geometrical scattering amplitude, and $\phi_{m}(\theta)$ is its phase. This phase represents the advance or retardation of the ray scattered in the $\theta$ direction with respect to the central ray

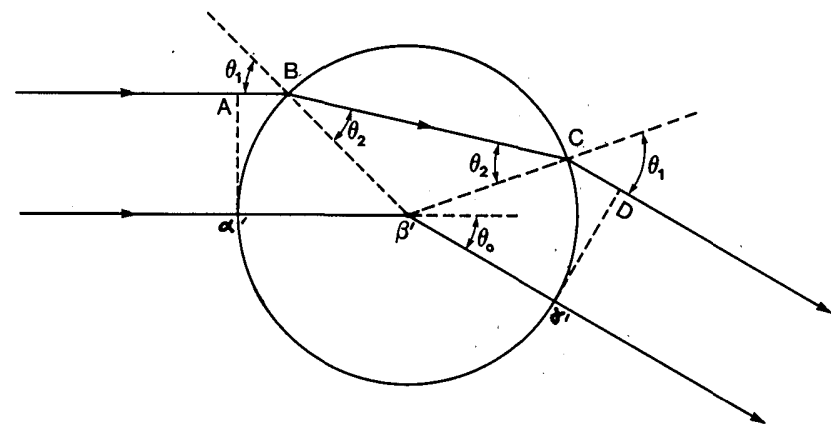

Fig. 2. Extra optical length of the transmitted ray $A B C D$ with respect to the central ray $\alpha^{\prime} \beta^{\prime} \gamma^{\prime}$ is $2 n a \cos \theta_{2}-2 a \cos \theta_{1}$, where $a$ is the droplet radius. This produces the phase retardation $2 \beta\left(n \cos \theta_{2}-\right.$ $\cos \theta_{1}$ ) with respect to the phase of the central ray.

in the absence of the sphere as is shown in Fig. 2. The phase $\phi_{m}(\theta)$ is given by ${ }^{21-23}$

$$
\phi_{m}(\theta)= \begin{cases}-2 \beta \sin (\theta / 2) & \text { for } m=0, \\ 2 \beta\left(m n \cos \theta_{2},-\cos \theta_{1}\right) & \text { for } m \geq 1,\end{cases}
$$

where $n$ is the index of refraction of the sphere,

$$
\beta=(2 \pi a) / \lambda,
$$

and $\theta_{1}$ and $\theta_{2}$ are the angles of incidence and refraction of the ray exiting in the $\theta$ direction, which are related by Snell's law:

$$
n \sin \theta_{2}=\sin \theta_{1} .
$$

If a single water droplet is observed at a close distance, the contribution of the geometrical scattering amplitude of order $m$ to the field observed at the retina is

$$
\begin{aligned}
\mathbf{E}_{r}^{(m)}(p)= & \int_{-\theta_{w / 2}}^{\theta_{w / 2}} d \xi \mathbf{A}_{m}\left(\theta_{0}+\xi\right) \\
& \times \exp \left[i \phi_{m}\left(\theta_{0}+\xi\right)\right] \exp (-i p \xi) .
\end{aligned}
$$

If the integrand is Taylor series expanded in the inter$\operatorname{val} \theta_{0}-\theta_{w / 2} \leq \theta \leq \theta_{0}+\theta_{w / 2}$, if $\mathbf{A}_{m}(\theta)$ is slowly varying and $\theta_{w}$ is small enough so that

$$
\frac{\theta_{w}^{3}}{48}\left[\frac{d^{3}}{d \theta^{3}} \phi_{m}(\theta)\right]_{\theta_{0}} \ll 1
$$

then

$$
\begin{aligned}
\mathbf{E}_{r}^{(m)}(p) \approx & \mathbf{A}_{m}\left(\theta_{0}\right) \exp \left[i \phi_{m}\left(\theta_{0}\right)\right] \int_{-\dot{\theta}_{w / 2}}^{\theta_{w / 2}} d \xi \\
& \times \exp \left[i \phi_{m}^{\prime \prime}\left(\theta_{0}\right) \xi^{2} / 2\right] \exp \left\{i\left[\phi_{m}^{\prime}\left(\theta_{0}\right)-p\right] \xi\right\},
\end{aligned}
$$

or

$$
\begin{aligned}
\left|\mathbf{E}_{r}^{(m)}(p)\right|^{2}= & \left|\mathbf{A}_{m}\left(\theta_{0}\right)\right|^{2} \frac{\pi}{\phi_{m}^{\prime \prime}\left(\theta_{0}\right)} \mid \mathscr{E}\left\{\frac{\phi_{m}^{\prime}\left(\theta_{0}\right)-p}{\left[\pi \phi_{m}^{\prime \prime}\left(\theta_{0}\right)\right]^{1 / 2}}+\frac{\theta_{w}}{2}\left[\frac{\phi_{m}^{\prime \prime}\left(\theta_{0}\right)}{\pi}\right]^{\mathrm{i} / 2}\right\} \\
& -\left.\mathscr{E}\left\{\frac{\phi_{m}^{\prime}\left(\theta_{0}\right)-p}{\left[\pi \phi_{m}^{\prime \prime}\left(\theta_{0}\right)\right]^{1 / 2}}-\frac{\theta_{w}}{2}\left[\frac{\phi_{m}^{\prime \prime}\left(\theta_{0}\right)}{\pi}\right]^{1 / 2}\right\}\right|^{2},
\end{aligned}
$$

where

$$
\phi_{m}^{\prime}\left(\theta_{0}\right)=\left[\frac{d}{d \theta} \phi_{m}(\theta)\right]_{\theta_{0}},
$$




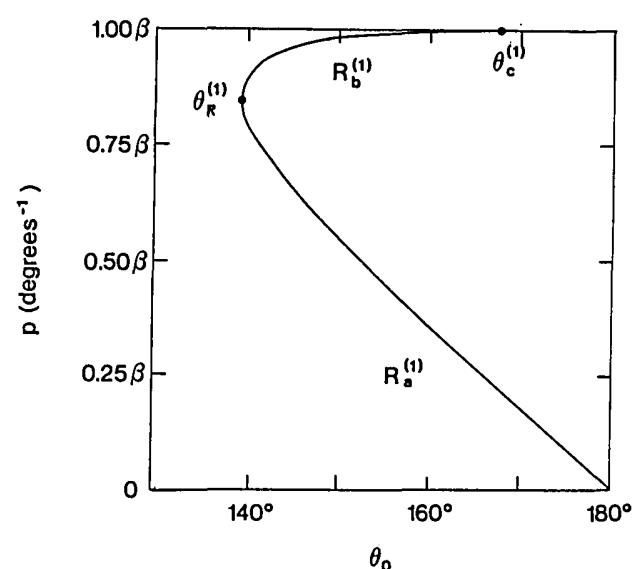

Fig. 3. Spatial frequencies of the first-order rainbow glare spots $R_{a}^{(1)}$ and $R_{b}^{(1)}$ as a function of the observation angle $\theta_{0} . \theta_{c}$ is the first rainbow critical angle of Eq. (26), and $\theta_{R}$ is the first-order rainbow angle of Eq. (28).

$$
\phi_{m}^{\prime \prime}\left(\theta_{0}\right)=\left[\frac{d^{2}}{d \theta^{2}} \phi_{m}(\theta)\right] \theta_{0},
$$

and $\mathscr{E}(x)$ is the Fresnel integral

$$
\mathscr{E}(x)=\int_{0}^{x} d u \exp \left(i \pi u^{2} / 2\right) .
$$

The contribution of the geometrical rays to the observed field takes the form of a series of glare spots centered on the retinal positions

$$
p_{m}=\phi_{m}^{\prime}\left(\theta_{0}\right)
$$

for $m \geq 0$. The glare spots have the shapes of Fresnel diffraction patterns, and their intensities are proportional to $\left|\mathbf{A}_{m}\left(\theta_{0}\right)\right|^{2}$. By substituting Eq. (11) into Eq. (20), the reflected glare spot has the spatial frequency

$$
p_{0}=-\beta \cos \theta_{0} / 2 \text {, }
$$

and the transmitted and rainbow glare spots have the spatial frequencies

$$
p_{m}=\beta \sin \theta_{1},
$$

where $\theta_{1}$ is related to the total deflection angle $\theta$ by

$$
\theta=(m-1) \pi+2 \theta_{1}-2 m \sin ^{-1}\left(\frac{\sin \theta_{1}}{n}\right) .
$$

For the transmitted ray, Eq. (23) is quadratic in $\sin \theta_{1}$ and may be solved to give

$$
p_{1}=n \beta\left(\sin \theta_{0} / 2\right)\left(n^{2}+1-2 n \cos \theta_{0} / 2\right)^{-1 / 2} .
$$

For the various order rainbows, Eq. (23) must be solved numerically. For the first-order rainbow and an index of refraction of $n=1.343$, the numerical solution to Eq. (23) may be substituted into Eq. (22) to give the spatial frequencies of the rainbow glare spots shown in Fig. 3.

These spatial frequencies behave in the expected way. The reflected glare spot $G$ appears at the edge of the droplet, $p=-\beta$, for the forward-scattering geometry. As the observer moves around the droplet toward the backscattering geometry, the location of the glare spot moves toward the center of the droplet, $p=0$. At forward scattering, the transmitted glare spot appears at the center of the droplet. As the observer moves toward the scattering angle at which the edge rays are critically refracted within the droplet,

$$
\theta_{c}^{(T)}=2 \cos ^{-1}\left(\frac{1}{n}\right)
$$

the location of the glare spot moves toward the other edge of the droplet, $p=\beta$. As can be seen in Fig. 3 for the first rainbow, the glare spot $R_{a}^{(1)}$ appears at the center of the droplet in the backscattering geometry. As $\theta_{0}$ decreases, $R_{a}^{(1)}$ moves toward the $p=\beta$ edge of the droplet. At the first rainbow critical angle,

$$
\theta_{c}^{(1)}=2 \pi-4 \sin ^{-1}\left(\frac{1}{n}\right),
$$

the other glare spot $R_{b}^{(1)}$ appears at the $p=\beta$ edge. As $\theta_{0}$ further decreases, the two glare spots approach each other and merge at the spatial frequency

$$
p=\left(\frac{4-n^{2}}{3}\right)^{1 / 2} \beta
$$

at the first-order rainbow angle,

$$
\theta_{R}^{(1)}=\pi+2 \sin ^{-1}\left(\frac{4-n^{2}}{3}\right)^{1 / 2}-4 \sin ^{-1}\left(\frac{4-n^{2}}{3 n^{2}}\right)^{1 / 2} .
$$

The behavior of the $m \geq 3$ rainbow glare spots is similar. Since in most conditions the total scattering amplitude is dominated by the contribution provided by the geometric ray amplitude, the observations made on a single water droplet and the identification of the glare spots seen on it provide an intuitive way to judge which terms of Eq. (9) are most important in the approximation of the Mie scattered field at a given observation angle.

\section{Glare Spots and Mie Scattering Theory}

The Mie scattered fields and their Fourier transforms were calculated for the parameters

$$
\begin{aligned}
a & =0.75 \mathrm{~mm}, \\
n & =1.343, \\
\lambda & =0.42 \mu \mathrm{m} .
\end{aligned}
$$

The droplet radius is the same as that employed in the experimental observations of Walker. ${ }^{9}$ At the red end of the visible spectrum, the third- and fourth-order rainbows nearly overlap. At the blue end, they are separated by about $10^{\circ}$. Since the purpose of this calculation was to extract the third-order rainbow from the directly transmitted light intensity and the light reflected from the surface, the wavelength at the blue end of the visible spectrum given in Eq. (31) was employed. The angular interval

$$
\theta_{w}=4.6^{\circ}
$$

was chosen to ensure that Eq. (15) was satisfied and to ensure that the diffractive effects of $F_{s}(\alpha)$ in Eq. (4) were minimal.

The Mie intensity ${ }^{24}$ in the vicinity of the third-order rainbow for initially unpolarized light is given in Fig. 4. The Fourier transform spectra ${ }^{24}$ of $\left|\mathbf{E}_{r}(p)\right|^{2}$ for a num- 


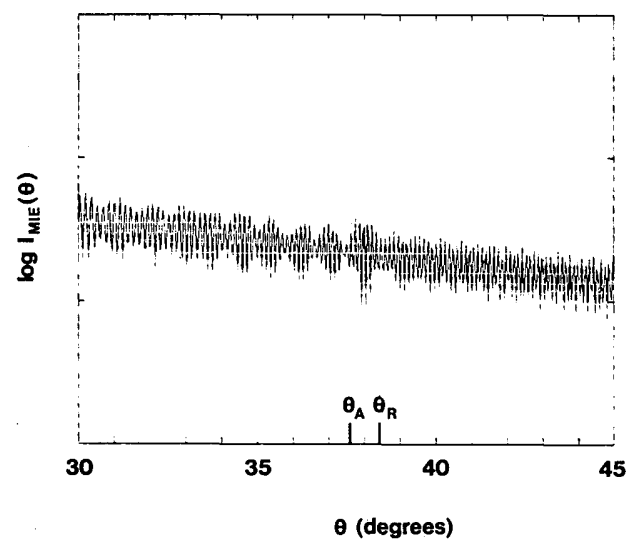

Fig. 4. Logarithm of the Mie intensity as a function of scattering angle in the vicinity of the third-order rainbow. $\theta_{A}$ and $\theta_{R}$ correspond to the Airy rainbow angle and the geometric rainbow angle, respectively.

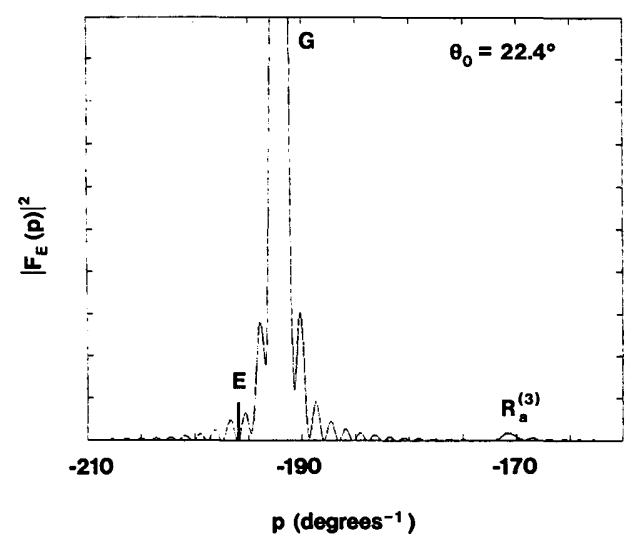

Fig. 5. Magnitude squared of the Fourier transform of the Mie electric field as a function of spatial frequency for the observation angle $\theta_{0}=22.4^{\circ}$. The edge of the drop $E$ is at $-195.83 \mathrm{deg}^{-1}$. $G$ and $R_{a}^{(3)}$ are predicted to be at -192.10 and $-170.66 \mathrm{deg}^{-1}$, respectively.

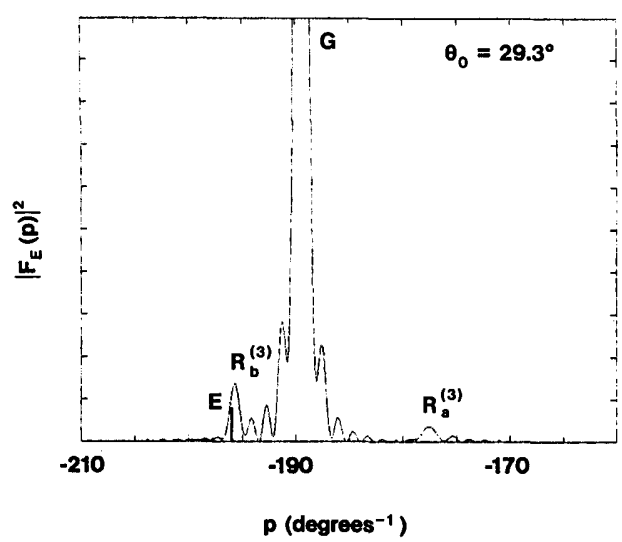

Fig. 6. Magnitude squared of the Fourier transform of the Mie electric field for $\theta_{0}=29.3^{\circ} . G, R_{a}^{(3)}$, and $R_{b}^{(3)}$ are predicted to be at $-189.46,-177.24$, and $-195.66 \mathrm{deg}^{-1}$, respectively.

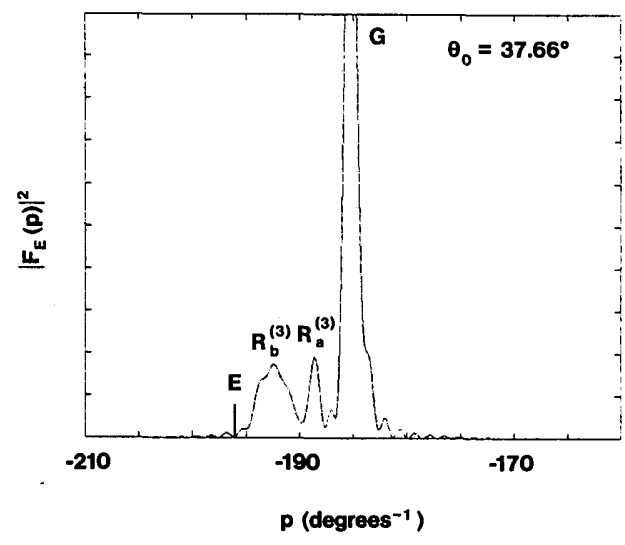

Fig. 7. Magnitude squared of the Fourier transform of the Mie electric field at the Airy rainbow angle $\theta_{0}=37.66^{\circ}$. According to the geometrical model, $G, R_{a}^{(3)}$ and $R_{b}^{(3)}$ are predicted to be at -185.35 , -188.29 , and $-192.32 \mathrm{deg}^{-1}$, respectively.

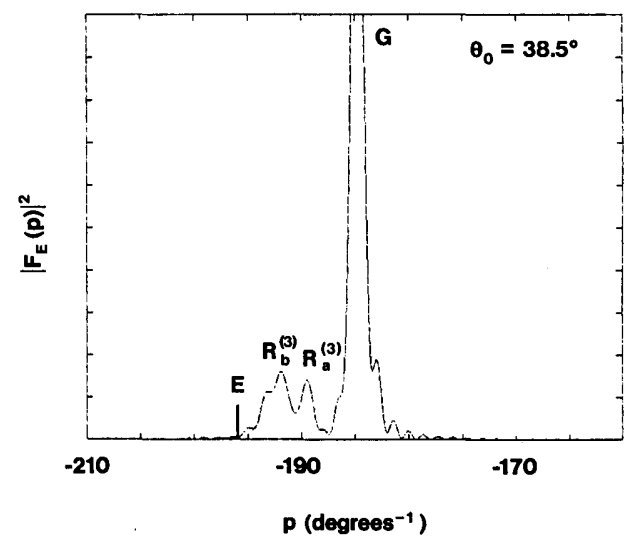

Fig. 8. Magnitude squared of the Fourier transform of the Mie electric field at $\theta_{0}=38.5^{\circ}$. The lower limit of the angular interval of Eq. (1) begins to approach the Airy angle, and as a result the $R_{a}^{(3)}$ and $R_{b}^{(3)}$ peaks begin to merge.

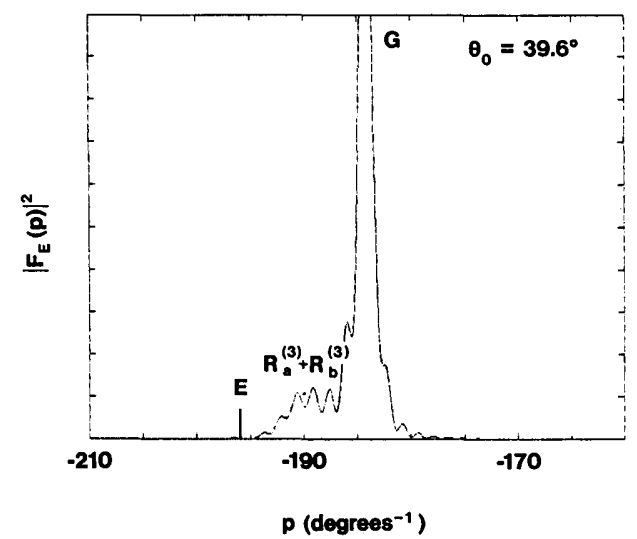

Fig. 9. Magnitude squared of the Fourier transform of the Mie electric field at $\theta_{0}=39.6^{\circ}$. The lower limit of the angular interval of Eq. (1) is nearly at the Airy angle, and as a result the merging of $R_{a}^{(3)}$ and $R_{b}^{(3)}$ peaks is complete. 


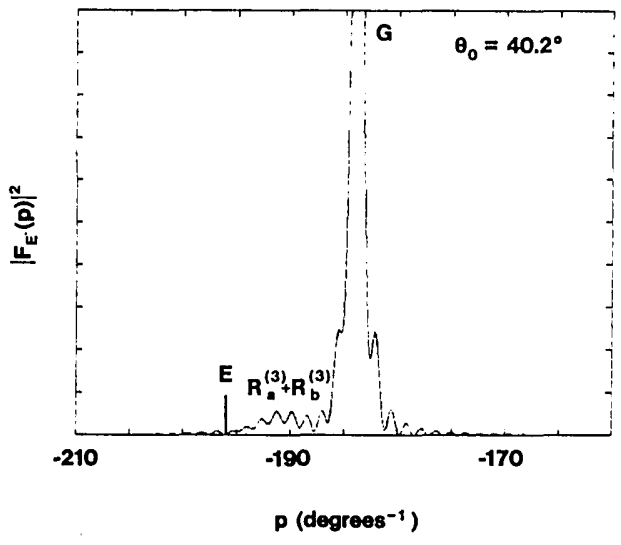

Fig. 10. Magnitude squared of the Fourier transform of the Mie electric field at $\theta_{0}=40.2^{\circ}$. The lower limit of the angular interval of Eq. (1) is between the Airy angle and geometrical rainbow angle. As a result, the rainbow peak loses strength.

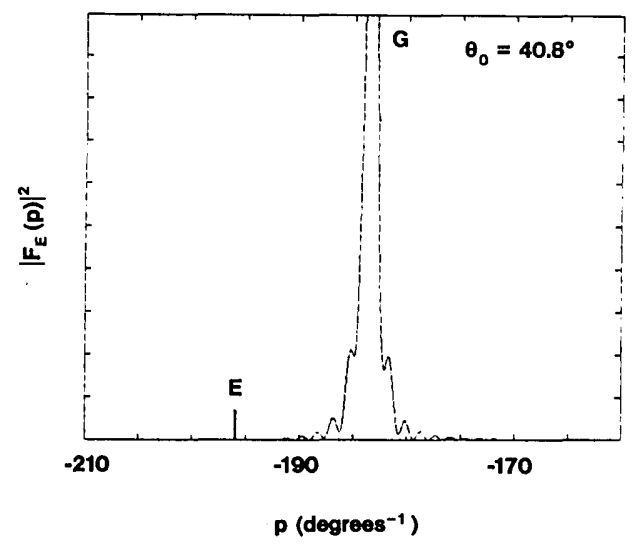

Fig. 11. Magnitude squared of the Fourier transform of the Mie electric field at $\theta_{0}=40.8^{\circ}$. $G$ is predicted to be at $-183.55 \mathrm{deg}^{-1}$.

ber of angles $\theta_{0}$ in the vicinity of the third-order rainbow are given in Figs. 5-11. In each of these figures the edge of the sphere $E$ corresponds to the spatial frequency $p=-\beta=-195.83 \mathrm{deg}^{-1}$. The third rainbow critical angle is $\theta_{c}^{(3)}=25.00^{\circ}$. Thus for $\theta_{0}=22.4^{\circ}$, Fig. 5 shows only the reflected glare spot $G$ and the single third rainbow glare spot $R_{a}^{(3)}$, while at $\theta_{0}=29.3^{\circ}$, Fig. 6 shows $G$ and both $R_{a}^{(3)}$ and $R_{b}^{(3)}$. The geometrical third-order rainbow angle is $\theta_{R}^{(3)}=38.11^{\circ}$, and the distorted wavefront Airy theory predicts that it is shifted down to $\theta_{A}^{(3)}=37.66^{\circ}$. Figures $7-10$ show the Fourier transform spectra for the sequence of angles 37.66, 38.5, 39.6, and $40.2^{\circ}$. Even though Fig. 7 is centered at the Airy angle, the $R_{a}^{(3)}$ and $R_{b}^{(3)}$ peaks have not merged since the Mie fields that contribute to this spectrum extend over the interval $35.36^{\circ} \leq \theta \leq 39.96^{\circ}$, and half of this angular interval is in advance of the predicted merging. Figures $8-10$ on the other hand extend over the intervals $36.2^{\circ} \leq \theta \leq 40.8^{\circ}, 37.3^{\circ} \leq \theta \leq$ $41.9^{\circ}$, and $37.9^{\circ} \leq \theta \leq 42.5^{\circ}$, respectively. In Figs. 8 and 9 , since the lower end of the angular interval approaches the Airy angle, the $R_{a}^{(3)}$ and $R_{b}^{(3)}$ peaks

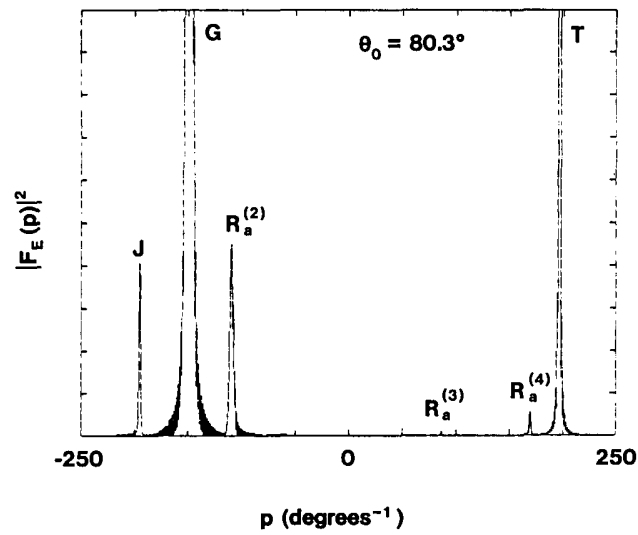

Fig. 12. Magnitude squared of the Fourier transform of the Mie electric field at $\theta_{0}=80.3^{\circ} . G, T, R_{a}^{(2)}, R_{a}^{(3)}, R_{a}^{(4)}$ are the reflected, transmitted, and second, third, and fourth rainbow glare spots, respectively. $J$ is the glare spot not attributable to geometric optics.

completely merge. Since the lower end of the angular interval of Fig. 10 is between the Airy angle and the geometric rainbow angle, the combined $R_{a}^{(3)}+R_{b}^{(3)}$ peak loses strength. Well past the rainbow angle in the shadow region, Fig. 11 for $\theta_{0}=40.8^{\circ}$ shows only the reflected glare peak. Similar behaviors were found in the vicinities of the first-order and second-order rainbows as well.

There is a curious feature of the Mie Fourier spectra that appears only near the critical angle for the $m=1$ transmitted ray or near the critical angles for the $m \geq 2$ rainbow rays. According to Eq. (25) for $n=1.343$, the critical angle for transmission occurs at $\theta_{c}^{(T)}=83.75^{\circ}$. The Fourier spectrum for $\theta_{0}=80.3^{\circ}$, just to the lit side of the critically refracted ray, is given in Fig. 12. In this figure, the glare spots $G, T, R_{a}^{(2)}, R_{a}^{(3)}$, and $R_{a}^{(4)}$ appear at the locations predicted by the geometrical amplitude. The geometrical rays producing these glare spots at $\theta_{0}=80.3^{\circ}$ are indicated in Figs. 13 and 14 . In addition, a weak glare spot $J$ that is unexplained in terms of geometric optics occurs at the $p=-\beta$ edge of the droplet in Fig. 12. It is found to occur only for angles $\theta_{0}$ near the critical angle. On deleting various partial waves from the Mie series, it was found that this nongeometrical peak, as well as the $T$ glare spot which it appears in conjunction with, is produced by the partial waves corresponding to the rays which graze the edge of the sphere. Similar nongeometrical peaks were numerically found near the first-, second-, and third-order rainbow critical angles as well. In every case they occur on the side of the droplet opposite to the corresponding critically refracted geometrical ray. If the nongeometrical peak were the Fourier transform of an appropriate scattering amplitude, the phase of that amplitude would have been

$$
\phi_{m}^{\text {nongeometric }}(\theta)=-2 \beta\left(m n \cos \theta_{2}-\cos \theta_{1}\right),
$$

representing a phase advance rather than the usual phase delay associated with the $m \geq 1$ refracting rays. The path of such a phase advanced ray is shown in Fig. 15. However, the physical mechanism responsible for 


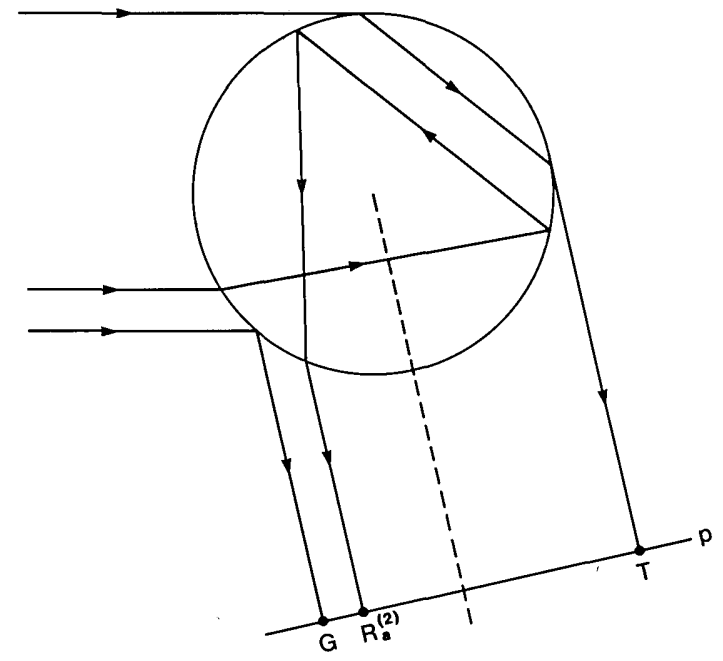

Fig. 13. Geometric rays which produce $G, T$ and $R_{a}^{(2)}$ at $\theta_{0}=80.3^{\circ}$ in Fig. 12.

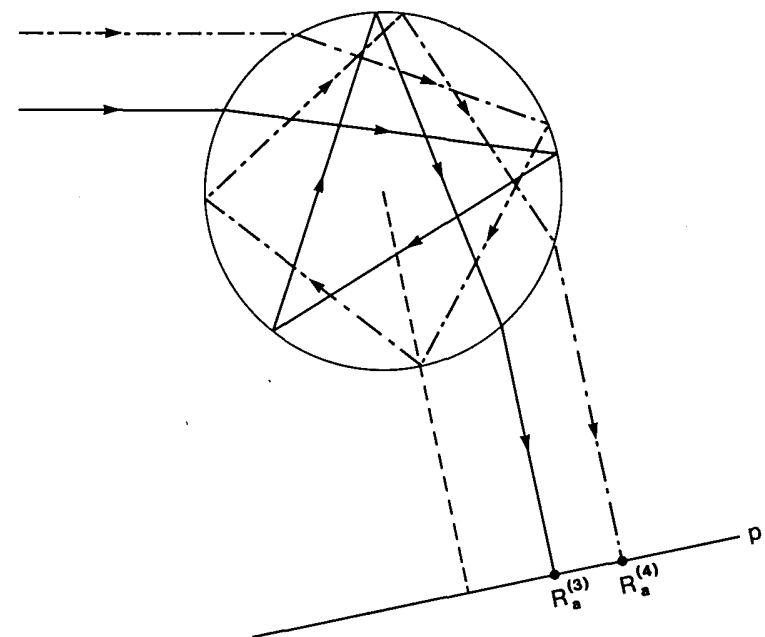

Fig. 14. Geometric rays which produce $R_{a}^{(3)}$ and $R_{a}^{(4)}$ at $\theta_{0}=80.3^{\circ}$ in Fig. 12.

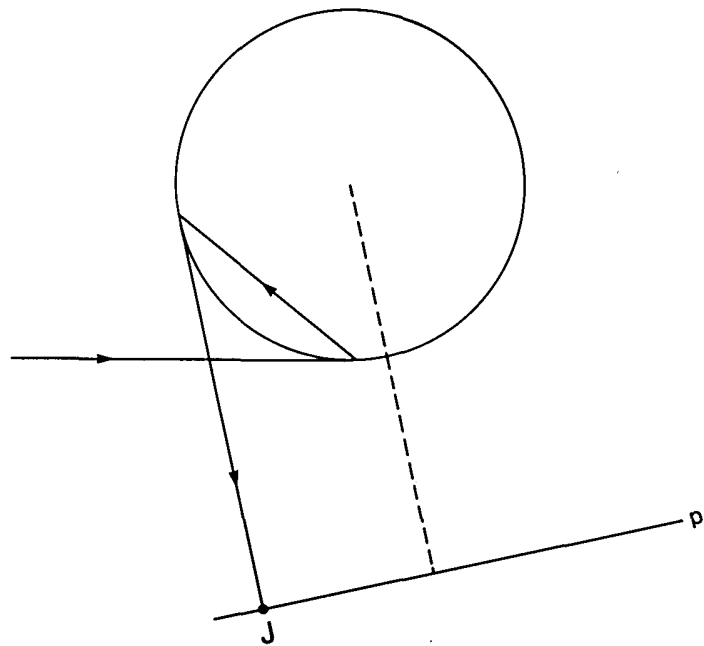

Fig. 15. Possible nongeometric critically refracted ray which produces the glare spot $J$ in Fig. 12. the $J$ peak of Fig. 12 is presently unclear since the nongeometric contribution to the scattering amplitude in the complex angular momentum analysis, the first and fourth quadrant residue poles, correspond to surface waves circulating around the droplet in the same sense as the grazing incident ray. ${ }^{25}$

\section{Conclusions}

The Mie infinite series of partial waves is the exact solution to the problem of the scattering of plane electromagnetic waves from a sphere. Being the exact solution, all the scattering effects that can possibly occur are hidden somewhere or another in the Mie amplitude. The big problem has always been that it is hard to extract information out of it since the Mie series is not expressed in a simple or transparent form. It is pleasing to see in this case that the experimental observations on a single water droplet can be understood by Fourier transforming the Mie fields. This reflects the fact that if many signals, each with its own periodicity, are superposed in the spatial domain, they become separated in the Fourier domain. The complex angular momentum theory used in conjunction with the Mie results helps to determine the extent to which the behavior of the glare spots is determined by geometrical considerations and the extent to which other considerations may contribute.

\section{References}

1. Accounts of the observation of third-order rainbows are given in C. B. Boyer, The Rainbow, from Myth to Mathematics (Thomas Yoseloff, New York, 1959), p. 271; a recent account is D. E. Pedgley, "A Tertiary Rainbow," Weather 41, 401 (1986).

2. R. A. R. Tricker, Introduction to Meterological Optics (American-Elsevier, New York, 1970), p. 57.

3. R. Greenler, Rainbows, Halos, and Glories (Cambridge, U. P., London, 1980), pp. 6 and 7.

4. C. W. Querfeld, "Mie Atmospheric Optics," J. Opt. Soc. Am. 55, 105 (1965).

5. J. V. Dave, "Scattering of Visible Light by Large Water Spheres," Appl. Opt. 8, 155 (1969).

6. K.-N. Liou and J. E. Hansen, "Intensity and Polarization for Single Scattering by Polydisperse Spheres: a Comparison of Ray Optics and Mie Theory," J. Atmos. Sci. 28, 995 (1971).

7. In regard to the observability of high-order rainbows in the atmosphere, Ref. 4 predicts that the blue component of the fifthorder rainbow should lie in Alexander's band for scattering from spherical water droplets and should possibly be visible.

8. The observability of high-order rainbows in nonspherical droplets is examined in K. Sassen, "Angular Scattering and Rainbow Formation in Pendant Drops," J. Opt. Soc. Am. 69, 1083 (1979).

9. J. Walker, "Multiple Rainbows from Single Drops of Water and Other Liquids," Am. J. Phys. 44, 421 (1976).

10. J. Walker, "How to Create and Observe a Dozen Rainbows in a Single Drop of Water," Sci. Am. 237, 138 (July 1977).

11. J. Walker, "Mysteries of Rainbows, Notably their Rare Supernumerary Arcs," Sci. Am. 242, 174 (June 1980).

12. Photographs of the rainbow glare spots are given in Ref. 2, pp. 44-46 and in Ref. 10. Many diagrams of the rainbow glare spots are also given in Refs. 9 and 10.

13. Drawings of these caustics appear many places in the literature. Among them are H. M. Nussenzveig, "The Theory of the Rainbow,"Sci. Am. 236, 116 (Apr. 1977); Ref. 1, Figs. 61-62; and W. J. 
Humphreys, Physics of the Air (Dover, New York, 1964), Fig. 174.

14. W. J. Humphreys, Physics of the Air (Dover, New York, 1964), pp. 484-492.

15. Sassen ${ }^{8}$ conjectures that the visibility of high-order rainbows is further enhanced for nonspherical droplets.

16. A. C. Holland and J. S. Draper, "Analytical and Experimental Investigation of Light Scattering from Polydispersions of Mie Particles," Appl. Opt. 6, 511 (1967).

17. J. W. Goodman, Introduction to Fourier Optics (McGraw-Hill, New York, 1968), p. 10

18. D. D. Cooke and M. Kerker, "Response Calculations for LightScattering Aerosol Particle Counters," Appl. Opt. 14, 734 (1975).

19. S. I. Rubinow, "Scattering from a Penetrable Sphere at Short Wavelengths," Ann. Phys. NY 14, 305 (1961).

20. H. M. Nussenzvieg, "High-Frequency Scattering by a Transpar- ent Sphere I. Direct Reflection and Transmission," J. Math. Phys. 10, 82 (1969).

21. H. M. Nussenzvieg, "High-Frequency Scattering by a Transparent Sphere II. 'Theory of the Rainbow and the Glory," J. Math. Phys. 10, 125 (1969).

22. V. Khare and H. M. Nussenzvieg, "Theory of the Rainbow," Phys. Rev. Lett. 33, 976 (1974).

23. H. M. Nussenzveig, "Complex Angular Momentum Theory of the Rainbow and the Glory," J. Opt. Soc. Am. 69, 1068 (1979).

24. The Mie series was summed via the method described in W. J. Wiscombe, "Improved Mie Scattering Algorithms," Appl. Opt. 19, 1505 (1980). Since the spatial frequencies for an FFT of these data were too widely spaced, the Fourier transform was performed directly via numerical integration of Eq. (1).

25. D. Ludwig, "Diffraction by a Circular Cavity," J. Math. Phys. 11, 1617 (1970).
Patter continued from page 5290

This work was done by Alan D. Clarke of United Technologies Corp. for Ames Research Center. Inquiries concerning rights for the commercial use of this invention should be addressed to the Patent Counsel, Ames Research Center. Refer to ARC-11585.

\section{Portable slot-sizing tool}

A portable milling tool consisting of an air-motor-driven cutter held in an adjustable moving slide made possible the local removal of chromium plating in a close-tolerance onsite remachining and sizing of half-hole slots on longeron bridges (see Fig. 13). Sending the bridges back to the manufacturer for rework would have been considerably more expensive, in addition to introducing project delays. An adaptation of the portable sizing tool could be useful for the field modification of such large equipment as trucks, aircraft, and ships, which are not easily returnable to factories. The tool is made from

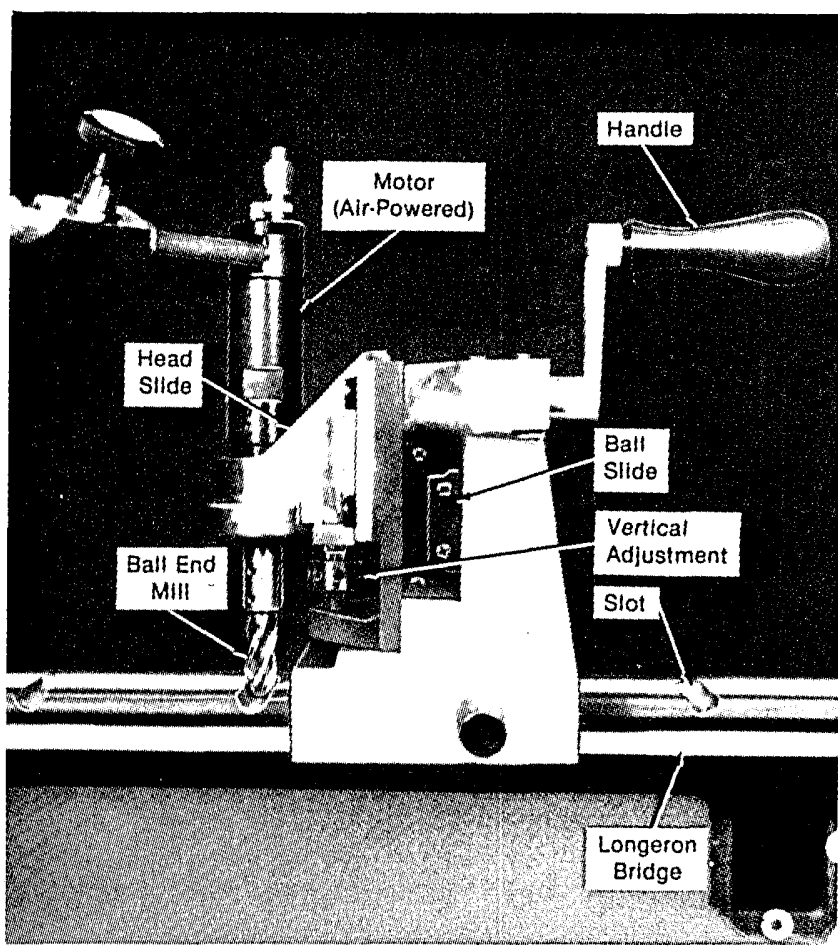

Fig. 13. This portable slot-sizing tool is shown being used to remachine and size half-hole slots on a longeron bridge. commercially available parts, including an air motor capable of variable speeds up to $900 \mathrm{rpm}$, a ball end mill, a revolving handle, two miter gears, and a ball slide. The tool is clamped to the longeronbridge rail, and the cutter is lined up with a given slot, approximately at the midpoint of the hole length. After the cutter is lowered to the proper depth, the motor is turned on and the crank handle used to move the cutter along the groove to remove the chromium, thereby restoring the slot to its specified dimensions.

This work was done by Nelson T. Zuver of Rockwell International Corp. for Johnson Space Center. Refer to MSC-21088.

\section{Grips for lightweight tensile specimens}

A set of grips has been developed for the tensile testing of lightweight composite materials (Fig. 14). Resin-based fiber-reinforced composite materials are more susceptible than metal specimens of comparable cross section to bending and twisting during installation and to slippage and deformation in the grip region. Even a small degree of misalignment in the specimen during tensile testing can affect the measured mechanical properties. The new grips minimize specimen misalignment and reduce scatter in the data. The gripping force is applied by driving hardened wedges against the end tabs of the specimen. This double wedging action, actually a wedge driven against another wedge, allows a greater force to be applied over a larger area, thereby minimizing slippage and deformation in the grip region. Alignment is maintained by assembling the grips inside a backplate on a horizontal surface. This backplate keeps the specimen from twisting and bending during installation. It is removed only after the assembly has been put firmly in place on the testing machine.

More than 75 specimens of 5 different lightweight materials, typically demonstrating fractures occurring evenly along the specimen

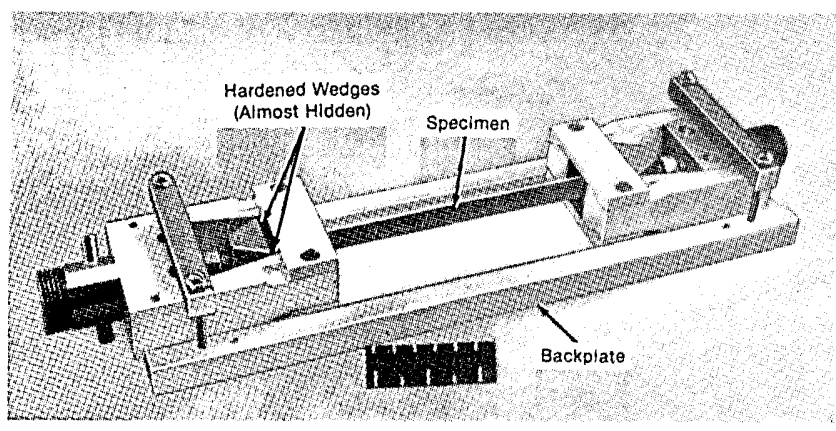

Fig. 14. Specimen is held by grips made of hardened wedges. The assembly is screwed into a load cell in a tensile-testing machine.

continued on page 5325 